\title{
Effects of container shape on seedling growth of Hevea brasiliensis
}

\author{
Chen Xianhong ${ }^{1}$, Wang Jun², Lin Weifu ${ }^{3 *}$
}

\author{
${ }^{1}$ Rubber Research Institute, Chinese Academy of Tropical Agricultural Sciences, Danzhou, Hainan 571737, China \\ E-mail: chenxhongjane@163.com \\ ${ }^{2}$ Rubber Research Institute, Chinese Academy of Tropical Agricultural Sciences, Danzhou, Hainan 571737, China \\ E-mail: wangjuncatas@163.com \\ ${ }^{3 *}$ Rubber Research Institute, Chinese Academy of Tropical Agricultural Sciences, Danzhou, Hainan 571737, China \\ E-mail: RubberL@163.com
}

\begin{abstract}
Effects of container shape on seedling growth of Hevea brasiliensis at different growth stages were observed to provide reference for rubber nursing. Rubber seedlings were planted in different shape containers with same height and volume. Then leaf whorl, stem diameter, plant height, tap root length, amount of first lateral root, root weight, shoot weight and root-shoot ratio were investigated at 90, 180 and 360 days after transplantation, respectively. The results showed that the optimal selection of container shape is the prism at 90 days, while the cube at 180 and 360 days. At 90 days, leaf whorls and root weight in prisms were significantly 10.3\%, 11.3\% more than in cylinders, plant height 13.4\%, 20.6\% higher than in cubes and cuboids, shoot weight 67.1\%, 58.8\%, $14.5 \%$ heavier than in cubes, cuboids and cylinders, respectively. At 180 days, stem diameter in cubes was significantly $16.4 \%, 10.9 \%$ bigger than in cuboids and prisms, plant height 16.5\% higher than in cuboids, tap root length $18.5 \%$ longer than in cylinders, numbers of first lateral root 22\%, 25\%, 56.5\% more than in cylinders, prisms and cuboids, shoot weight 23.5\%, 40.4\% and root weight 19.9\%, 19.5\% heavier than in prisms and cuboids, respectively. At 360 days, plant height in cubes was significantly $11.7 \%$ higher than in prisms and tap root length $14.7 \%$ longer than in cylinders. Based on specific surface area of containers and the growth at all days, the optimal selection of container shape is the cube and then the cylinder.
\end{abstract}

Keywords-Container shape, Hevea brasiliensis, seedlings, growth.

\section{INTRODUCTION}

Rubber seedling production in China mainly uses plastic bags to raise seedlings and nursery for 6-22 months, which usually causes seedling roots twisted, and some serious recover slowly after planting, resulting in irregular rubber forest phase. Mauer et al. investigated the relationship between root distribution, degree of deformity and growth of aboveground parts in 90 container seedlings plantations in Czech Republic, which showed that the adverse effects of root deformity caused by cylindrical container seedlings on growth could not disappear until 15 years, the adverse effects of peat and bottomless container could disappear after 10 years, root malformations of polyethylene bags and Koparfors porous container seedlings remained unchanged after 20 years of afforestation (Deng, 2008). Therefore, it is of great significance to study the effect of container shape on the root system and growth of rubber seedlings.

Container shape has great influence on root growth and development of Pinus tabulaeformis seedlings (Gao, 1983) and Picea spruce seedlings (Lesterw et al. ,1988). Container size has significant effects on seedling height, ground diameter and biomass of Quercus suber L. seedlings (Chirino et al., 2008), Pingyi sweet tea seedlings (An et al., 2013), Castanea mollissima grafted seedlings (Tan, 2016), Osmanthus fragrans seedlings (Qiu et al., 2018), Chipi Qinggang (Wang et al., 2019). The growth of Platycladus orientalis (Dong et al., 2006), Fraxinus mandshurica seedlings (Wei et al., 2016) and one-year-old white wax (Du 
et al., 2019) in non-woven bags were significantly better than that in plastic containers. Different ratio of nursery substrate components also have a significant impact plant height, ground diameter and biomass in container seedlings of Manglietia grandis (Zheng et al., 2018), Osmanthus fragrans seedlings (Qiu et al., 2018), Parashorea chinesis container seedlings (Pang et al., 2018), Millennium Tung container seedlings (Hong, 2019), Pinus tabulaeformis (Dong et al., 2019).

At present, container seedling research mainly focuses on nursery substrate, container material and sizes, but there are few studies on container shape of seedling which are based on experience and lack of relevant theoretical basis. This study is aimed at comparing the growth of rubber seedlings planted in containers of different shapes at different stages to understand the effect of container shape on the root system and growth of rubber seedlings and further recommend suitable rubber seedling container shape.

\section{MATERIALS AND METHODS}

\subsection{Experimental materials}

Rubber seedlings of two weeks-old were assembled into air pruning containers (without base) with same height and volume and different shapes (cylinder, prism, cube and cuboid), which were made of recycled HDPE plastic. The specific surface area of the container was cuboid $>$ prism $>$ cube > cylinder, and the seedling-raising medium was coconut bran.

\subsection{Experimental method}

The experiment was conducted from May 2017 to May 2018 in the protective cultivation base of natural rubber of Rubber Research Institute of Chinese Academy of Tropical Agricultural Sciences, Danzhou City, Hainan Province, China. The coconut bran of culture medium was put into containers with the same volume and different shapes and placed at the same density. Rubber seedlings with the same growth from the sand bed were transplanted into the containers. In order to ensure that the roots were not penetrated, the containers were placed on the cement ground. The water, fertilizer and pesticide management were carried out accordingly. Numbers of leaf canopy, stem diameter, plant height, main root length, numbers of primary lateral roots, root weight, shoot weight and rootshoot ratio of each treatment were observed at 90, 180 and 360 days after transplantation, respectively. Ten plants were observed each time in three replicates. Vernier caliper $(0.02$ $\mathrm{mm})$ was used to measure stem diameter, and steel ruler $(0.5$ $\mathrm{mm}$ ) was used to measure plant height and main root length. After detaching the containers and shaking off the coconut bran, the whole seedlings were separated immediately. The shoot and root weights were measured by the analytical balance.

\subsection{Data analysis}

Statistical analyses were performed with Data Processing System (DPS) statistical software package version 16.5 (Tang, 2013) using one-way ANOVA followed by the Duncan's Multiple Range Test (SSR) to evaluate significant difference among seedlings from different container shapes at $\mathrm{P}<0.05$ and Topsis (technology for order preference by similarity to ideal solution) method for comprehensive evaluation analysis.

\section{RESULTS}

3.1 Effect of container shapes on the aboveground part growth of rubber seedlings

3.1.1 Effect on numbers of leaf whorls As shown in Fig.1, at 90 days, the number of leaf whorls of seedlings in prisms is $10.3 \%$ more than that in cylinders. There was no significant difference in the number of seedling leaf whorls among cylinders, cubes and cuboids, and among cubes, cuboids and prisms. At 180 days, the number of leaf whorls of seedlings in cuboids was significantly less than that in cylinders by $10 \%$ and there was no significant difference among cylinders, cubes and triangular prisms and among cuboids, cubes and prisms. At 360 days, the number of seedlings leaf whorls in prisms was $5.2 \%$ and $4.76 \%$ less than that in cubes and cuboids, respectively. There was no significant difference between the number of seedlings leaf whorls in cylinders and prisms, and among cylinders, cubes and cuboids. 

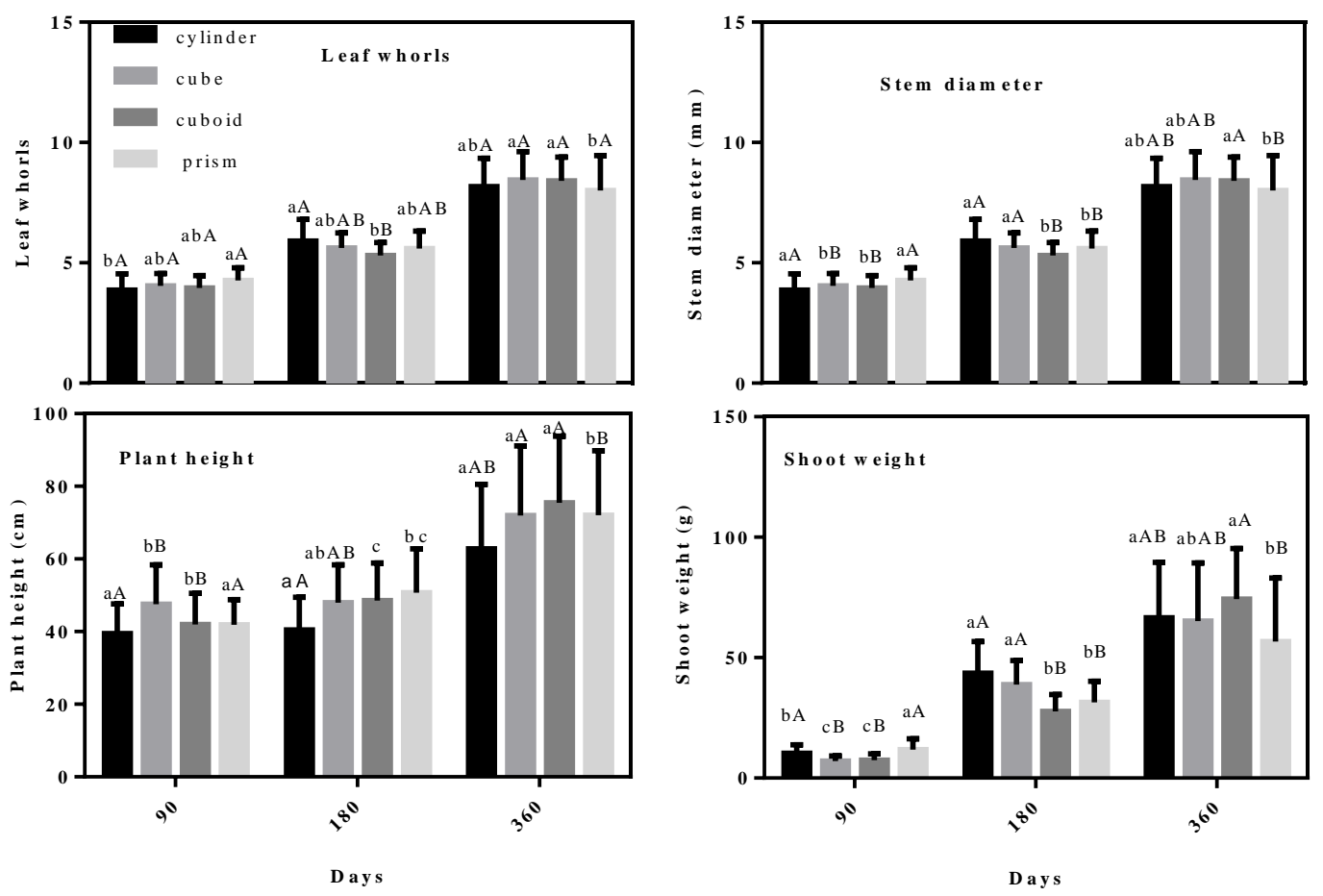

Fig.1 Comparison of leaf whorls, stem diameter, plant height, shoot weight of rubber seedlings raised in four container shapes at different growth days. Values are means $\pm S D(n=10)$. Different lowercase or capital letters indicate significant at 0.05 and 0.01, respectively. The same letter is not significantly different.

3.1.2 Effect on stem diameter As shown in Fig.1, at 90 days, stem diameter of cubes and cuboids was $13 \%$ and $10.3 \%$ smaller than that in cylinders, $15.7 \%$ and $13.1 \%$ smaller than that in prisms, respectively. There was no significant difference between stem diameter of seedlings in cylinders and prisms, and between stem diameter of seedlings in cuboids and cubes. At 180 days, the stem diameter of seedlings in cuboids was $13.7 \%$ and $14.1 \%$ smaller than those in cylinders and cubes, respectively. The stem diameter of seedlings in prisms was $9.4 \%$ and $9.8 \%$ smaller than those in cylinders and cubes, respectively. There was no significant difference between stem diameter of seedlings in cylinders and cube, and between stem diameter of seedlings in cuboids and prisms. At 360 days, there was no significant difference among stem diameter of seedlings in cylinders, cuboids and cubes. The stem diameter of seedlings in prisms was $11.2 \%$ smaller than that in cuboids. 3.1.3 Effect on plant height As shown in Fig.1, at 90 days, there was no significant difference between plant height of seedlings in cylinders and that in prisms, and between plant height of seedlings cuboids and cubes. The plant height of seedlings in prisms was $13.4 \%$ and $20.6 \%$ higher than those in cubes and cuboids, respectively. The plant height of seedlings in cubes and cuboids was significantly higher than that in cylinders by $14.5 \%$ and $8.5 \%$, respectively. At 180 days, the plant height of seedlings in cubes was $16.5 \%$ higher than that in cuboids; the plant height of seedlings in cuboids and prisms was $19 \%$ and $11.7 \%$ lower than that in cylinders, respectively; the plant height of seedlings in cylinders and cubes, cubes and prisms, cuboids and prisms, were not significant. At 360 days, there was no significant difference among plant height of seedlings in cylinders, cuboids and cubes. The height of seedlings in prisms was $7.8 \%, 10.5 \%$ and $12.1 \%$ smaller than those in cylinders, cubes and cuboids, respectively.

3.1.4 Effect on shoot weight As shown in Fig.1, at 90 days, shoot weight of seedlings in cube, cuboid and cylinder were $40.1 \%, 37 \%$ and $12.7 \%$ lighter than those in prisms, respectively. The shoot weight of seedlings in cubes and cuboids were $31.5 \%$ and $28 \%$ lighter than those in cylinders, respectively. There was no significant difference between shoot weight of seedlings in cuboids and cubes. At 180 days, 
there was no significant difference between shoot weight of seedlings in cylinders and cubes, and between shoot weight of seedlings in cuboids and prisms. The shoot weight of seedlings in cubes was $40.4 \%$ and $23.5 \%$ more than those in cuboids and prisms, and the shoot weight of seedlings in cuboids and prisms was $36.5 \%$ and $27.9 \%$ less than that of seedlings in cylinders, respectively. At 360 days, there was no significant difference among shoot weight of seedlings in cylinders, cuboids and cubes, and between shoot weight of seedlings in cubes and prisms. The shoot weight of seedlings in prisms was $14.8 \%$ and $23.6 \%$ lighter than those in cylinders and cuboids, respectively.

3.2 Effect of container shapes on the underground part growth of rubber seedlings

3.2.1 Effect on tap root length As shown in Fig.2, at 90 days, the tap root length of seedlings in cylinders, cuboids and prisms was significantly shorter than that of seedlings in cubes by $16.9 \%, 11.6 \%$ and $11.8 \%$, respectively, while there was no significant difference in the tap root length of seedlings in cylinders, cuboids and prisms. At 180 days, the tap root length of seedlings in cubes, cuboids and prisms was significantly shorter than that in cylinders by $18.5,20 \%$ and $25.3 \%$, respectively, while there was no significant difference among the tap root length of seedlings in cubes, cuboids and prisms. At 360 days, there was no significant difference among the tap root length of seedlings in cubes, cuboids and prisms, and the tap root length of seedlings in cubes, cuboids and prisms was significantly longer than that in cylinders by $14.7 \%, 20.2 \%$ and $14.8 \%$, respectively.
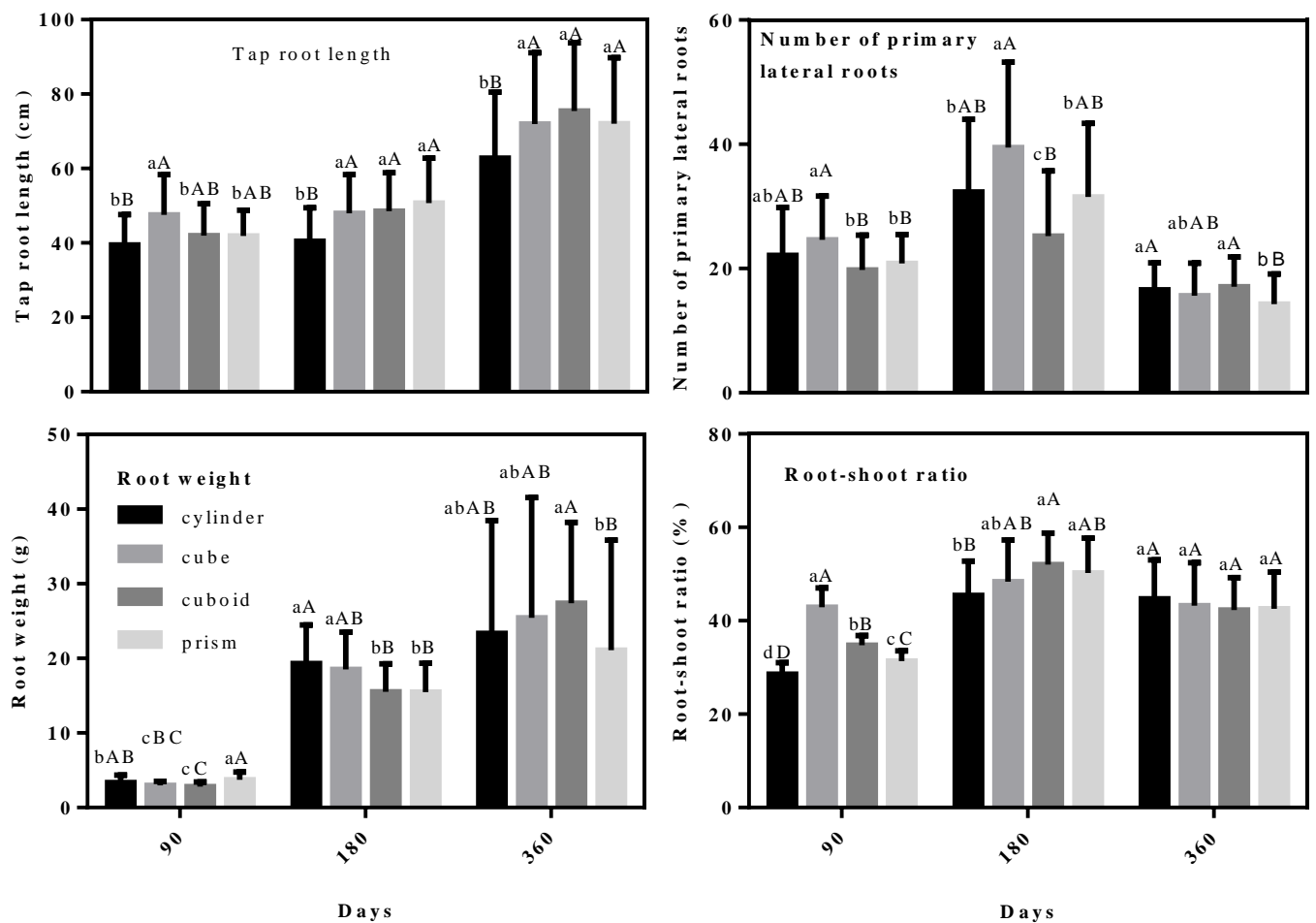

Fig.2 Comparison of tap root length, the first lateral root length, root weight, root and shoot ratio of rubber seedlings raised in four container shapes at different growth days. Values are means $\pm S D(n=10)$. Different lowercase or capital letters indicate significant at 0.05 and 0.01, respectively. The same letter is not significantly different.

\subsubsection{Effect on the number of primary lateral roots As} shown in Fig.2, at 90 days, the number of primary lateral roots of seedlings in prisms was not significantly different from that of seedlings in cylinders and cubes. The number of primary lateral roots of seedlings in prisms and cubes was not significantly different. The number of primary lateral roots of seedlings in prisms and cuboids was $15.4 \%$ and $19.7 \%$ less than that in cube, respectively. At 180 days, there was no significant difference in the number of primary lateral roots between the seedlings in cylinders and prisms. 
The number of primary lateral roots in the cube was $18 \%$ and $25 \%$ more than those in the cylinder and the prism, respectively. The number of primary lateral roots in the cuboids was $10 \%, 36.1 \%$ and $20 \%$ less than those in cylinders, cubes and prisms respectively. At 360 days, there was no significant difference among the number of primary lateral roots of seedlings in cylinders, cuboids and cubes, and between the number of primary lateral roots of seedlings in cubes and prisms. The number of primary lateral roots of seedlings in prisms was $13.8 \%$ and $16.4 \%$ less than those in cylinders and cuboids, respectively.

2.2.3 Effect on root weight As shown in Fig.2, at 90 days, the root weight of seedlings in prisms was $11.3 \%$ heavier than that in cylinders. The root weight of seedlings in cuboids and cubes was $15.5 \%$ and $11.6 \%$ lighter than that of seedlings in cylinders, $24.1 \%$ and $20.6 \%$ lighter than that of seedlings in prisms, respectively. There was no significant difference between root weight of seedlings in cuboids and cubes. At 180 days, there was no significant difference between the root weight of seedlings in cylinders and cubes, and between the root weight of seedlings in cuboids and prisms. The root weight of seedlings in prisms and cuboids was $20 \%$ and $19.8 \%$ lighter than that in cylinders, $16.6 \%$ and $16.4 \%$ lighter than that in cubes. At 360 days, the root weight of seedlings in cylinders, cuboids and cubes had no significant difference. The root weight of seedlings in cylinders, cubes and prisms had no significant difference. The root weight of seedlings in prisms was $23 \%$ lighter than that in cuboids.

\subsection{Effect of container shapes on root-shoot ratio}

As shown in Fig.2, at 90 days, the root-shoot ratios of seedlings in cuboids, cubes and prisms were 50.2\%, $21.9 \%$ and $9.8 \%$ more than that in cylinders, respectively. The rootshoot ratios of seedlings in cuboids and prisms were $18.9 \%$ and $26.9 \%$ less than that in cubes, respectively. The rootshoot ratios of seedlings in prisms were $9.9 \%$ less than that in cubes. At 180 days, the root-shoot ratio of seedlings in cylinders and cubes had no significant difference and the root-shoot ratio of seedlings in cubes, cuboids and prisms had no significant difference either. The root-shoot ratio of seedlings in cubes and prisms was $14.6 \%$ and $10.6 \%$ more than that in cylinders, respectively. At 360 days, there was no significant difference in root-shoot ratio of seedlings in cylinders, cubes, cuboids and prisms.

3.4 Comprehensive evaluation of the effect of container shape on the growth of rubber seedlings

Based on the specific surface area, aboveground and underground observation indexes, and the size of CI value (approximation to the optimal vector), the comprehensive evaluation results of different container shapes on the growth of rubber seedlings are shown in Table 1. The best container shape at 90 days is the prism, followed by the cylinder. At 180 days and 360 days, the best container shape is the cube, followed by the cylinder. Combining the specific surface area and the observation indexes of three growth periods (90 days, 180 days, 360 days), we find that the best container shape is the cube, followed by the cylinder.

Table 1 Comprehensive evaluation of different container shapes on rubber seedling growth

\begin{tabular}{cccccc}
\hline \multirow{2}{*}{ Days } & $\begin{array}{c}\text { Container } \\
\text { shapes }\end{array}$ & Cuboid & $\begin{array}{c}\text { Triangle } \\
\text { prism }\end{array}$ & Cube & Cylinder \\
\hline \multirow{4}{*}{90} & D+ & 0.3711 & 0.2271 & 0.312 & 0.255 \\
& D- & 0.1081 & 0.3249 & 0.2749 & 0.2763 \\
& CI & 0.2256 & 0.5886 & 0.4683 & 0.5201 \\
& Rank & 4 & 1 & 3 & 2 \\
& D+ & 0.3883 & 0.2714 & 0.1082 & 0.1765 \\
& D- & 0.1213 & 0.1804 & 0.3311 & 0.338 \\
& CI & 0.238 & 0.3994 & 0.7537 & 0.657 \\
& Rank & 4 & 3 & 1 & 2 \\
& D+ & 0.2154 & 0.1995 & 0.1189 & 0.157 \\
& D- & 0.1946 & 0.1729 & 0.1816 & 0.1928
\end{tabular}




\begin{tabular}{cccccc} 
& CI & 0.4746 & 0.4644 & 0.6043 & 0.5513 \\
360 & Rank & 3 & 4 & 1 & 2 \\
& D+ & 0.5177 & 0.4049 & 0.341 & 0.3423 \\
& D- & 0.2855 & 0.377 & 0.4443 & 0.4261 \\
Total & CI & 0.3554 & 0.4822 & 0.5658 & 0.5545 \\
& Rank & 4 & 3 & 1 & 2 \\
\hline
\end{tabular}

D+, distance to optimal vector. D-, distance to inferior vector. CI, approximation to the Optimal Vectors.

\section{DISCUSSION}

The container shape has a great influence on the growth of seedlings. Growth tends to be stimulated when there is a mutual matching between the natural growth pattern of roots and the shape of their container (Biran, I and Eliassaf, A,1980). In this study, according to the aboveground and underground observation indexes, it is suggested that the prism container should be selected first for rubber seedling propagation at 90 days, the cube container at 180 days and 360 days, followed by the cylinder. The container shape has a greater impact on the spruce seedling (Lesterw et al. 1988), which suggested that the use of different shape containers should be flexible according to specific needs. The root system of the container seedlings of Pinus tabulaeformis with 60 degree prism is the best, followed by the regular triangle prism and the cylinder (Gao, 1983). It is further confirmed that the shape of the container has a great influence on the seedlings growth. In this study, the effects of container shape on stem diameter, plant height and main root length of rubber seedlings varied with the growth time. Among them, the biggest stem diameter of rubber seedlings was found in prisms at 90 days, cylinders at 180 days, cubes at 360 days, respectively. The highest plant height was found in cubes at 90 days, prisms at 180 days and cuboids at 360 days, respectively. The tap root length of rubber seedlings was the longest in cubes at 90 days, prisms at 180 days and cuboids at 360 days, respectively. Gao (1983) found that container shape had no significant effect on the root diameter, seedling height and main root length of seedlings, which may be related to the time of observation, the shape of the container, and the genus of the seedlings.

\section{CONCLUSION}

In this study, when the height and volume of each container with different shape are the same, the specific surface area, the aboveground and underground observation indexes of rubber seedlings in the different shape containers are synthetically evaluated. At 90 days, the prism is preferred as the nursery container shape of the rubber seedlings, followed by the cylinder; at 180 days and 360 days, the cube is preferred, followed by the cylinder. Based on the comprehensive evaluation of the specific surface area of each nursery container shape, the aboveground and underground observation indexes in three growth periods, the cube was the first choice as the container shape to nursery rubber seedlings, followed by the cylinder.

\section{ACKNOWLEDGEMENTS}

This work was supported by the earmarked fund for China Agriculture Research System (CARS-34-YZ4).

\section{REFERENCES}

[1] An N N, Fan W G, Tan Q P, Yang J Q, Gao D S. 2013. Effects of the different container sizes and its transition on growth of Malus Hupehensis Rehd seedlings. Journal of Shandong Agricultural University (Natural Science), 44(01):12-17.

[2] Biran I, Eliassaf A. (1980). The effect of container shape on the development of roots and canopy of woody plants. Scientia Horticulturae, 12(2), 183-193. 12(2), 183-193.

[3] Chirino E, Vilagrosa A, Hernández EI, Matos A, Vallejo VR. (2008). Effects of a deep container on morpho-functional characteristics and root colonization in Quercus suber L. seedlings for reforestation in Mediterranean climate. Forest Ecology and Management, 256(4), 779-785

[4] Deng H P. 2008. Container nursery technique of forest tree[M]. China Agriculture Press. Beijing. P31

[5] Dong J, Han Y Z, Li J J, Song L. 2019. Effects of different matrix compositions on the growth status of Pinus Tabulae Formis container seedlings. Journal of Shanxi Agricultural Sciences, 47(04): 624-627.

[6] Dong Z C, Wang Y H, Zhou S H, Wang S H, Zhan H Q. 2006. Comparision of Platycladus Orientalis seedlings raised 
in polybags and non-woven fabric container. Shandong Forestry Science and Technology, (03): 35-36.

[7] Du H B, Wang X F, Liu Y Q, Li Z C, Xu Z Y. 2019. Effects of container types on Fraxinus container seedlings in light culture media Shandong forestry science and technology, 49(01): 43-44.

[8] Gao Y J. 1983.Effects of container shapes on the seedling growth and development. Shanxi forestry science and technology, (02): 14-20.

[9] Hong Y. 2019. Effects of different proportion matrixes to the growth of Vernicia Montana container seedling.Forestry and environmental science, 35(01): 89-93.

[10] Lesterw. Calson, Frankendena, Dai C Y, Song Z. 1988. Effects of roots and container shape on the early growth of Picea Glauca seedlings. The Journal of Hebei Forestry Science and Technology, (04):43-46

[11] Pang S H, Ma Y, Zhang P, Lao Q X, Yang B G, Liu S L. 2018. Effects of substrate ratio and applying dosages of slowrelease fertilizer on the growth of container Parashorea Chinesis seedlings. Journal of Northwest Forestry University. 33(06):66-71.

[12] Qiu Q, Yang D J, Zhong P, Liu J M, Chen Y. 2018. Effects of Seedling Substrate, Container Size and Fertilizer Amount on the Seedling Growth of Paramichelia baillonii. Journal of West China Forestry Science. 47(06):131-135.

[13] Tan L M 2016. Study on nurse seedling graft and anvil container seedlings technology in Castanea Henryi[D] p37.

[14] Tang, QY and Zhang, C X. 2013. Data Processing System (DPS) software with experimental design, statistical analysis and data mining developed for use in entomological research. Insect Science. 20(2): 254-260.

[15] Wang X H, Zhang D B, Wu X L, Chu X L, Zhou Z C. 2019. Effects of container size and nutrition loading on the seedling growth of precious tree species. Journal of Northwest Forestry University. 34(3):118-124.

[16] Wei X, Lu L, Li G Y, Tang YY. 2016. Effects of air-cutting on seedling growth and root development of fraxinus mandshurica in non-waven bags. Scientia Silvae Sinicae.52(09): 133-138.

[17] Zheng K Y, Kang L, Guo M Q, Liu T T, Luo Y Y, Deng X M. 2018. Effect of different substrate compositions on growth of container seedlings of Manglietia grandis. South China Forestry Science.46(06): 12-16. 